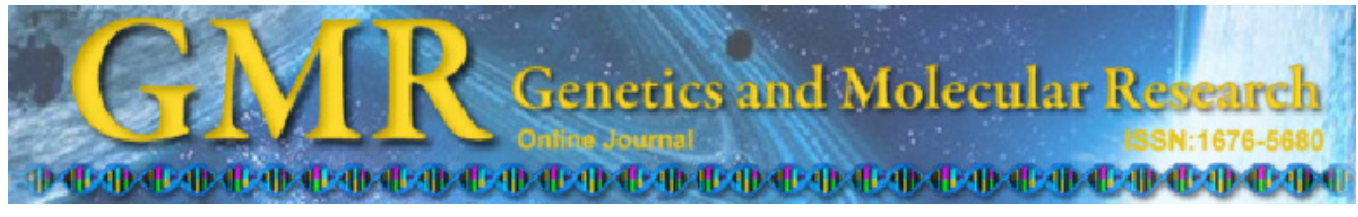

\title{
Detection of novel SNPs and mapping of the fatness QTL on pig chromosome 7q1.1-1.4 region
}

\author{
W.H. Huang, Z.X. Ma, Z.Y. Xu, Y.Z. Xiong and B. Zuo
}

Key Laboratory of Swine Genetics and Breeding, Ministry of Agriculture, Key Lab of Agricultural Animal Genetics and Breeding, Ministry of Education, College of Animal Science and Veterinary Medicine, Huazhong Agricultural University, Wuhan, P.R. China

Corresponding author: B. Zuo

E-mail: zuobo@mail.hzau.edu.cn

Genet. Mol. Res. 10 (4): 3090-3097 (2011)

Received January 13, 2011

Accepted April 20, 2011

Published December 14, 2011

DOI http://dx.doi.org/10.4238/2011.December.14.2

\begin{abstract}
Many QTLs for fatness traits have been mapped on pig chromosome 7q1.1-1.4 in various pig resource populations. Eight novel markers, including seven SNPs and one insertion or deletion within BTNL1, COL21A1, PPARD, GLP1R, MDFI, GNMT, ABCC10, and $P L A 2 G 7$ genes, as well as two previously reported SNPs in $S L C 39 A 7$ and $H M G A 1$ genes, were genotyped in Large White and Meishan pig breeds. Except for two SNPs in $H M G A 1$ and $A B C C 10$ genes, allele frequencies of the other eight markers are highly significant different between Chinese indigenous Meishan breeds and Large White pig breeds. Eight polymorphic sites were then used for linkage and QTL mapping to refine the fatness QTL in a Large White $\times$ Meishan $\mathrm{F}_{2}$ resource population. Five chromosome-wise significant QTLs were detected, of which the QTLs for leaf fat weight, backfat thickness at 6-7th rib and rump, and mean backfat thickness were narrowed to the interval between PPARD and GLPIR genes and the QTL for backfat thickness at thorax-waist between GNMT and PLA2G7 genes on SSC7p1.1-q1.4.
\end{abstract}

Key words: Single nucleotide polymorphism; Quantitative trait locus; Carcass traits; Sus scrofa chromosome 7 (SSC7) 


\section{INTRODUCTION}

To date, about 95 quantitative trait loci (QTL) related to fat deposition traits have been described on Sus scrofa chromosome 7 (SSC7) according to PigQTLdb (http://www. animalgenome.org/QTLdb/pig.html). We previously mapped significant QTL for backfat thickness and leaf fat weight between Sw1856 and Sw859 on SSC7 using a Large White x Meishan $F_{2}$ resource population (Zuo et al., 2004). It is interesting to note that the regions containing the QTL for fat deposition are in good agreement with the many results reported in various Meishan-derived populations (Milan et al., 1998; Moser et al., 1998; Rohrer and Keele, 1998; de Koning et al., 1999, 2001; Rohrer, 2000; Rattink et al., 2000; Bidanel et al., 2001; Sato et al., 2003; Demeure et al., 2005). Nearly all the QTL were located in the p1.1-q1.4 region, homologous to the short arm of human chromosome 6 (HSA6p), where QTL for some obesity indices in humans reside (Norman et al., 1995), and the allele with higher backfat thickness originated from the commercial breed and not from the Meishan pigs. Therefore, there is a strong possibility that the same cryptic QTL exist in the Meishan population and more research should be conducted to explore this possibility. Improving the genetic marker resolution of the linkage map in this QTL region would facilitate the search for QTL candidate genes. Single nucleotide polymorphism (SNP) maps are becoming the gold standard for genetic markers, even for linkage analyses (Bellenguez et al., 2009). Recently, the Wellcome Trust Sanger Institute released a partial BAC-based assembly of the porcine genome (Sscrofa5), which includes assemblies for pig chromosome 7. The gene order and genomic sequence at SSC7p1.1-q1.4 region can be accessed through the website http://www.ncbi.nlm.nih.gov/projects/mapview/maps.cgi?taxid=9823\&chr=7. In this study, an SNP-based genetic linkage map was constructed and used for the refined localization of fatness QTL on this region.

\section{MATERIAL AND METHODS}

\section{Animals and fatness traits}

Two populations, including Large White and Chinese indigenous Meishan pigs, were used for allele frequency estimation. The Large White $\mathrm{x}$ Meishan resource population including $315 \mathrm{~F}_{2}, 54 \mathrm{~F}_{1}$ and 21 grandparent animals were used for the linkage and QTL analysis (Zuo et al., 2007; Yang et al., 2010). Three of the seven Large White and eight of the fourteen Meishan founders of the resource population came from the populations used for allele frequency estimation. The animals were born and raised in the Huazhong Agriculture University Jingpin pig farm. All pigs were fed twice daily with diets formulated according to age under the standardized feeding and management regimen, and given free access to water. The $\mathrm{F}_{2}$ pigs were slaughtered at an average of 200 days, with a slaughter weight of $87.0 \pm 7.07 \mathrm{~kg}$. The pigs were slaughtered following a common protocol (Xiong and Deng, 1999). The fatness traits included thickness at shoulder (BFT1, cm), backfat thickness at thorax-waist (BFT2, cm), backfat thickness at rump (BFT3, cm), backfat thickness at 6-7th rib (BFT4, cm), average backfat thickness at shoulder, thorax-waist and rump (ABF, cm), leaf fat weight (LFW, $\mathrm{kg}$ ), and caul fat weight $(\mathrm{kg})$. 


\section{SNP identification and genotyping}

The order of ten genes was shown on the BAC fingerprint contig from the Wellcome Trust Sanger Institute (http://www.ncbi.nlm.nih.gov/projects/mapview/maps.cgi?taxid= $9823 \& \mathrm{chr}=7$ ). According to this map, the genomic region on SSC7q1.1-1.4 spans approximately $20 \mathrm{Mb}$, and the corresponding human region contains about 160 annotated genes. Based on gene information, ten genes (BTNL1, SLC39A7, COL21A1, HMGA1, PPARD, GLP1R, MDFI, GNMT, ABCC10, and PLA2G7) were selected. Two SNPs in SLC39A7 and HMGA1 genes were reported in the literature (Kim et al., 2006; Chen et al., 2009). The other SNPs were identified by comparative DNA sequencing of four Large White and four Meishan pigs and then confirmed using PCR or PCR-restriction fragment length polymorphisms (RFLP). Sequencing was done by a commercial service (Biotech Company, Beijing, China). Nucleotide sequences of all primers, annealing temperatures, allele pattern, and restriction endonucleases of eight makers are shown in Table 1. Animal genotyping was performed by PCR or PCR-RFLP with the application of the restriction endonucleases and separated on agarose gel or polyacrylamide gel.

\section{Linkage and QTL mapping analysis}

Linkage analysis was performed using CRIMAP version 2.4 (Green et al., 1990). Least square regression interval mapping was used for QTL detection (Haley et al., 1994). QTL analysis was carried out on internet (http://www.xmarks.com/site/qtl.cap.ed.ac.uk/). Using multi-marker information, three probabilities were calculated at 1-cM intervals along the chromosome. $\mathrm{P}_{(\mathrm{QQ})}, \mathrm{P}_{(\mathrm{qq})}$ and $\mathrm{P}_{(\mathrm{Qq})}$ are the probability that $\mathrm{F}_{2}$ offspring inherited two Large White alleles, two Meishan alleles and one from each breed, respectively. At every centimorgan (cM) across the genome, the following model was fitted:

$$
y_{i j k}=u+s_{i}+f_{j}+\beta c o v_{i j k}+c_{a k} a+c_{d k} d+e_{i j k},
$$

where $y_{i j k}$ is the trait record of the $\mathrm{k}^{\text {th }}$ offsping; $u$ is the overall mean; $s_{i}$ is the $\mathrm{i}^{\text {th }}$ sex effect $(i=1$, $2) ; f_{j}$ is the full-sib family $(j=1-37)$. Weight and age at slaughter were used as the covariate for carcass traits and meat quality traits, respectively. $a$ and $d$ are the estimated additive and the dominance effects of a putative QTL, respectively. $c_{a k}$ is the additive coefficient of the $\mathrm{k}^{\text {th }}$ individual at a putative QTL position and the probability $1 / 2\left(\mathrm{P}_{(\mathrm{QQ})}-\mathrm{P}_{(\mathrm{qq})}\right) \cdot c_{d k}$ is the dominant coefficient of the $\mathrm{k}^{\text {th }}$ individual at a putative QTL position and the probability $\mathrm{P}_{(\mathrm{Oq})} \cdot e_{i j k}$ is the residual. In this study, additive effects are estimated for the Large White QTL allele. Thus, positive values of the additive effects denote an increase of the trait due to the Large White QTL allele. Chromosome-wise significant thresholds are obtained by 1000 iterations of permutation (Churchill and Doerge, 1994). The confidence intervals of QTL were estimated by a bootstrap method with 1000 iterations (Visscher et al., 1996). The percentage of phenotype variances explained by QTL $\left(\mathrm{h}^{2}{ }_{\mathrm{Q}}\right)$ is calculated using the formula below:

$$
\mathrm{h}_{\mathrm{Q}}^{2}=\left(\mathrm{MS}_{\text {reducel }}-\mathrm{MS}_{\text {full }}\right) / \mathrm{MS}_{\text {reduce }} \times 100 \%,
$$

where $M S_{\text {full }}, M S_{\text {reducel }}$ and $M S_{\text {reduce }}$ are residual mean squares of the model with all detected QTL, the rest detected QTL except for a given one, and without QTL, respectively (Liu et al., 2009). 


\section{RESULTS AND DISCUSSION}

Except for two SNPs in SLC39A7 and HMGA1 genes, eight novel markers including seven SNPs and one insertion or deletion within BTNL1, COL21A1, PPARD, GLP1R, MDFI, GNMT, $A B C C 10$, and PLA2G7 genes, respectively, were identified and confirmed in this study. The 10 polymorphic sites were genotyped in Large White and Meishan pigs and allele frequencies were estimated (Table 2). The $\chi^{2}$ test showed that allele frequencies of the other markers were highly significantly different between Chinese indigenous Meishan and Large White pig breeds except for two SNPs in HMGA1 and ABCC10 genes. Due to the genotypic distribution skewing and the resulting low polymorphic information contents of two SNPs in $H M G A 1$ and $A B C C 10$ genes in the resource family, these two substitutions were excluded from further analysis. As a result, eight polymorphic sites were used for linkage mapping in a three-generation Large White x Meishan resource family. Mendelian segregation of the polymorphisms was observed in the resource family. The most probable order and position of DNA markers are shown in Table 1. The sex-averaged linkage map spanned $24.6 \mathrm{cM}$, with an average interval of $3.51 \mathrm{cM}$. The QTL mapping results are presented in Table 3. In this study, a total of five chromosome-wise significant QTL were detected (Figure 1).

The most significant QTL was that affecting BFT2 at position $23 \mathrm{cM}$ between GNMT and PLA2G7 $(\mathrm{P}<0.05)$. The QTL for BFT2 could explain approximately a $5.207 \%$ fraction of the phenotypic variation. Individuals homozygous for the Large White alleles were on average $0.396 \mathrm{~cm}$ thicker than those homozygous for Meishan alleles, and the Large White allele caused an increase in BFT2. The significant QTL for BFT3, BFT4, ABF and LFW were all mapped to the same marker interval between PPARD and GLP1R. These facts indicated that the overlapping QTL for these fatness traits could be caused by the same major gene. Three QTL for BFT3, ABF and BFT4 explaining, respectively, 4.999, 3.975 and $4.668 \%$ of the genetic variance were found with high significance at positions 11 and $12 \mathrm{cM}$ on SSC7q1.1-1.4. The negative additive estimate of BFT3, BFT4 and ABF QTL indicated higher backfat thickness for the Large White allele compared to the allele of the Meishan breed. The QTL for LFW was detected at position $11 \mathrm{cM}$ explaining $4.575 \%$ of total genetic variance, with the Meishan allele decreasing the phenotypic value. For all the QTL detected in this study, the favorable allele decreasing the fat deposition originated from the Meishan breed, which was contrary to the breed characteristic difference in fatness traits, but it confirmed many previous QTL effects according to PigQTLdb (Hu et al., 2005). Moreover, a two-QTL model analysis was performed to confirm if there were two QTL on this region, but no significant evidence for the existence of two QTL versus one QTL was observed. The order of 10 genes was also shown on the BAC fingerprint contig from the Wellcome Trust Sanger Institute. From this map, the new refined fatness QTL region from PPARD to GLP1R spans approximately $3.5 \mathrm{Mb}$, and the corresponding human region contains at least 28 annotated genes. Thus, the most probable position of fatness QTL between PPARD and GLP1R was located within a region of about $3.5 \mathrm{Mb}$ including 28 putative porcine genes. Several functional genes have been demonstrated to play important roles in the regulation of adiposity, including PPARD, CLPS and GLP1R genes, which could be considered as possible positional candidates. Meta-analysis of QTL in mice showed that PPARD and CLPS were all contained in one prominent region with linkage for body weight and body fat on mouse chromosome 17 (Wuschke et al., 2007). Therefore, a more comprehensive genetic and functional characterization of the candidate genes including 
W.H. Huang et al.

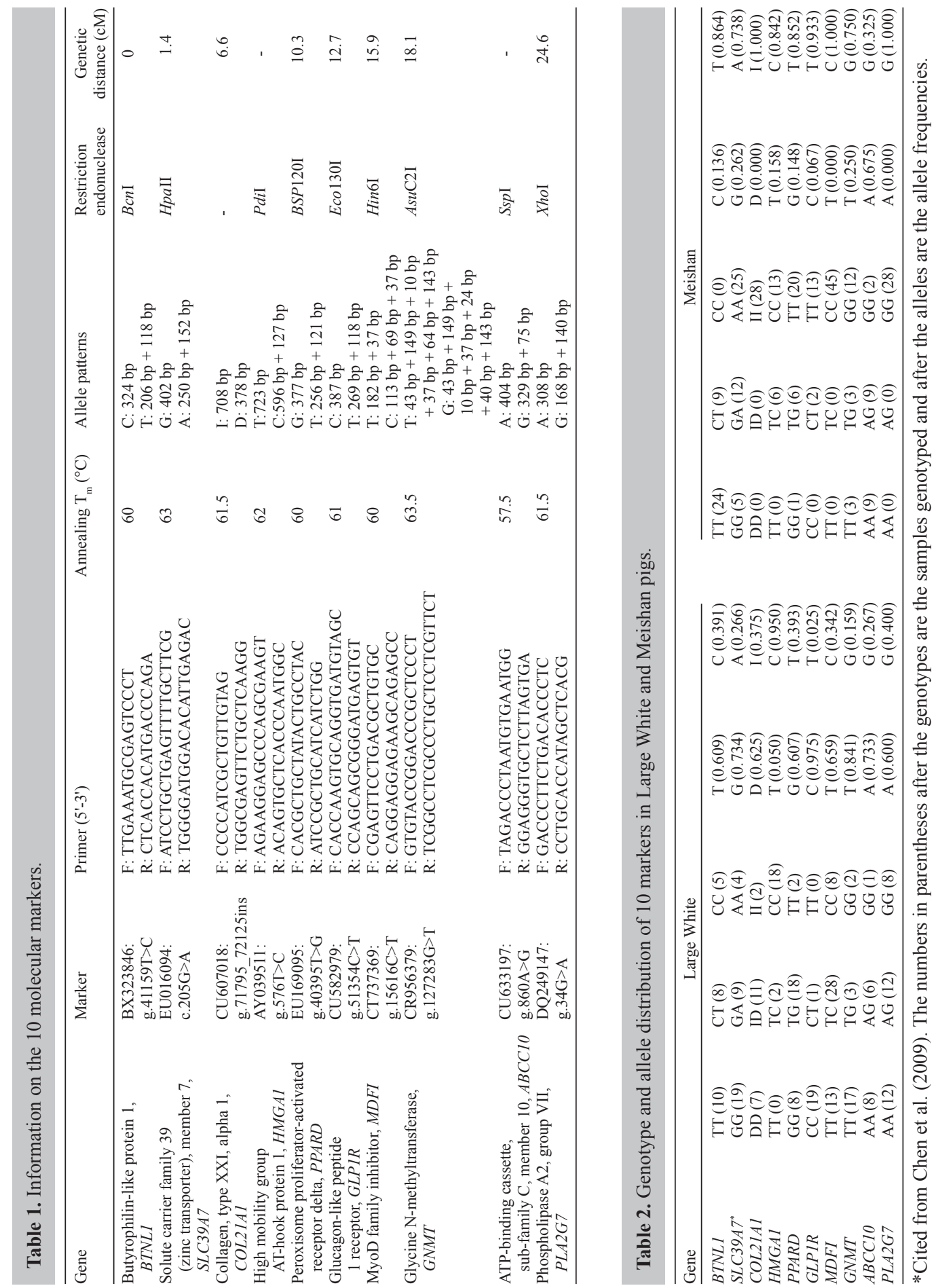

Genetics and Molecular Research 10 (4): 3090-3097 (2011) 
Table 3. QTL analysis for the fatness traits analyzed.

\begin{tabular}{lcclcccc}
\hline Traits & Position $(\mathrm{cM})$ & F-statistic & Marker interval & $\mathrm{a} \pm \mathrm{SEM}$ & $\mathrm{d} \pm \mathrm{SEM}$ & $\mathrm{h}^{2}{ }_{\mathrm{Q}}$ & Chromosome-wise significance level \\
\hline LFW & 11 & 7.63 & PPARD-GLPIR & $-0.085 \pm 0.022$ & $0.024 \pm 0.029$ & 4.575 & $<0.01$ \\
CFW & 20 & 3.44 & GNMT-PLA2G7 & $-0.023 \pm 0.025$ & $0.099 \pm 0.040$ & 1.709 & - \\
BFT1 & 11 & 3.76 & PPARD-GLPIR & $-0.191 \pm 0.075$ & $-0.051 \pm 0.099$ & 1.965 & - \\
BFT2 & 23 & 8.56 & GNMT-PLA2G7 & $-0.198 \pm 0.048$ & $-0.042 \pm 0.080$ & 5.207 & $<0.01$ \\
BFT3 & 11 & 8.18 & PPARD-GLPIR & $-0.225 \pm 0.061$ & $-0.078 \pm 0.081$ & 4.999 & $<0.01$ \\
BFT4 & 12 & 7.60 & PPARD-GLPIR & $-0.209 \pm 0.057$ & $-0.045 \pm 0.075$ & 4.668 & $<0.01$ \\
ABF & 11 & 6.71 & PPARD-GLPIR & $-0.186 \pm 0.055$ & $-0.056 \pm 0.073$ & 3.975 & $<0.05$ \\
\hline
\end{tabular}

LFW = leaf fat weight; CFW = caul fat weight; BFT1, 2, 3, 4 = backfat thickness at shoulder, thorax-waist, rump, and 6-7th rib, respectively; $\mathrm{ABF}=$ average backfat thickness at shoulder, thorax-waist and rump. $\mathrm{a}=$ additive effect; $\mathrm{d}=$ dominance effect; $\mathrm{SEM}=$ standard error; $\mathrm{h}_{\mathrm{O}}{ }_{\mathrm{O}}=$ fraction of phenotypic variance explained by the QTL. Negative values of the additive effects denote a decrease of the trait due to the Meishan alleles.
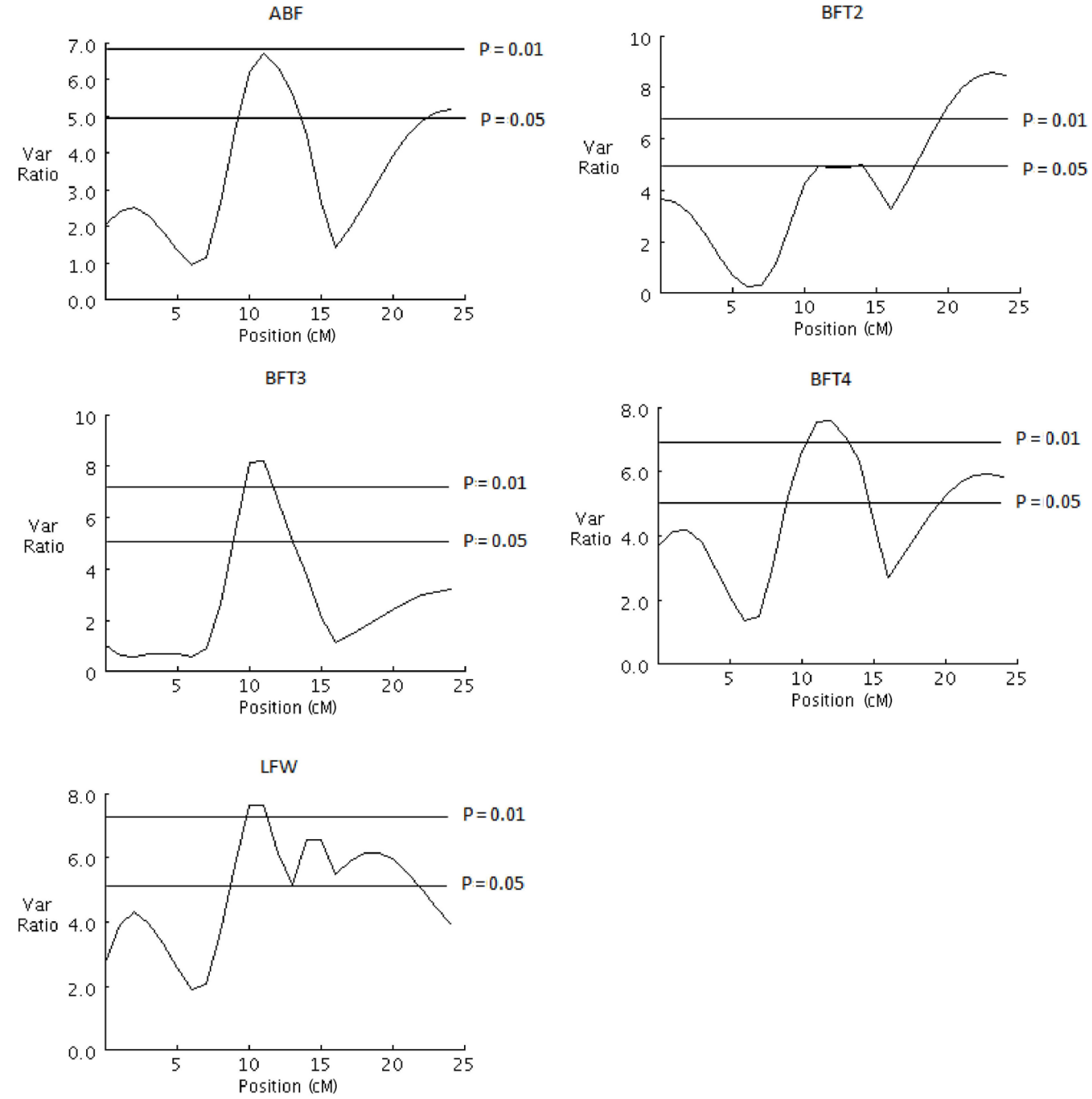

Figure 1. QTL mapping curve of fatness traits on SSC7 q1.1-1.4 region. The $\mathrm{x}$-axis indicates the relative position on the linkage map and the $y$-axis represents the F-ratio. 
expression analyses in target tissues should be performed to identify the gene responsible and ultimately the causative molecular genetic variation.

In summary, this study identified eight novel molecular markers and detected five significant QTL for fatness traits in a Large White $\mathrm{x}$ Meishan $\mathrm{F}_{2}$ resource population based on a new SNP linkage map. The results narrowed the previous QTL on SSC7p1.1-q1.4 and laid the basis for further comprehensive scanning of SNPs within the candidate functional genes.

\section{ACKNOWLEDGMENTS}

Research supported by the National Natural Science Foundation of P.R. China (\#31001043 and \#30500358), the National Major Special Projects of Science and Technology (\#2008ZX08006-002), the Key National High Technology Development Project (\#2011AA100301), the Agricultural Innovation Fund of Hubei Province, and the Creative Team Project of Education Ministry of China (\#IRT0831).

\section{REFERENCES}

Bellenguez C, Ober C and Bourgain C (2009). Linkage analysis with dense SNP maps in isolated populations. Hum. Hered. 68: 87-97.

Bidanel JP, Milan D, Iannuccelli N, Amigues Y, et al. (2001). Detection of quantitative trait loci for growth and fatness in pigs. Genet. Sel. Evol. 33: 289-309.

Chen ZG, Ma ZX, Zuo B, Lei MG, et al. (2009). Molecular characterization and association with carcass traits of the porcine SLC39A7 gene. J. Anim. Breed. Genet. 126: 288-295.

Churchill GA and Doerge RW (1994). Empirical threshold values for quantitative trait mapping. Genetics 138: 963-971.

de Koning DJ, Janss LL, Rattink AP, van Oers PA, et al. (1999). Detection of quantitative trait loci for backfat thickness and intramuscular fat content in pigs (Sus scrofa). Genetics 152: 1679-1690.

de Koning DJ, Rattink AP, Harlizius B and Groenen MA (2001). Detection and characterization of quantitative trait loci for growth and reproduction in pigs. Livest. Prod. Sci. 72: 185-198.

Demeure O, Sanchez MP, Riquet J, Iannuccelli N, et al. (2005). Exclusion of the swine leukocyte antigens as candidate region and reduction of the position interval for the Sus scrofa chromosome 7 QTL affecting growth and fatness. $J$. Anim. Sci. 83: 1979-1987.

Green P, Falls K and Crooks S (1990). Document for CRIMAP, Version 2.4. School of Medicine, Washington University, St. Louis.

Haley CS, Knott SA and Elsen JM (1994). Mapping quantitative trait loci in crosses between outbred lines using least squares. Genetics 136: 1195-1207.

Hu ZL, Dracheva S, Jang W, Maglott D, et al. (2005). A QTL resource and comparison tool for pigs: PigQTLDB. Mamm. Genome 16: 792-800.

Kim KS, Lee JJ, Shin HY, Choi BH, et al. (2006). Association of melanocortin 4 receptor (MC4R) and high mobility group AT-hook 1 (HMGA1) polymorphisms with pig growth and fat deposition traits. Anim. Genet. 37: 419-421.

Liu LT, Ren J, Li L, Li WB, et al. (2009). A genome scan for loci affecting adipocyte size and number in abdominal fat in a White Duroc x Erhualian $\mathrm{F}_{2}$ resource population. Animal 3: 632-636.

Milan D, Bidanal JP, Le Roy P, Le Roy P, et al. (1998). Current status of QTL detection in Large White x Meishan crosses in France. Proc. 6th World Congr. Genet. Appl. Livest. Prod. 26: 414-417.

Moser G, Mueller E, Beckmann P and Yue G (1998). Mapping of QTL in F2 generations of Wild Boar, Pietrain and Meishan pig. Proc. 6th World Congr. Genet. Appl. Livest. Prod. 26: 478-481.

Norman RA, Bogardus C and Ravussin E (1995). Linkage between obesity and a marker near the tumor necrosis factoralpha locus in Pima Indians. J. Clin. Invest. 96: 158-162.

Rattink AP, de Koning DJ, Faivre M, Harlizius B, et al. (2000). Fine mapping and imprinting analysis for fatness trait QTLs in pigs. Mamm. Genome 11: 656-661.

Rohrer GA (2000). Identification of quantitative trait loci affecting birth characters and accumulation of backfat and weight in a Meishan-White Composite resource population. J. Anim. Sci.78: 2547-2553.

Rohrer GA and Keele JW (1998). Identification of quantitative trait loci affecting carcass composition in swine: I. Fat 
deposition traits. J. Anim. Sci. 76: 2247-2254.

Sato S, Oyamada Y, Atsuji K, Nade T, et al. (2003). Quantitative trait loci analysis for growth and carcass traits in a Meishan x Duroc F2 resource population. J. Anim. Sci. 81: 2938-2949.

Visscher PM, Thompson R and Haley CS (1996). Confidence intervals in QTL mapping by bootstrapping. Genetics 143: 1013-1020.

Wuschke S, Dahm S, Schmidt C, Joost HG, et al. (2007). A meta-analysis of quantitative trait loci associated with body weight and adiposity in mice. Int. J. Obes. 31: 829-841.

Xiong YZ and Deng CY (1999). Principle and Method of Swine Testing. Chinese Agricultural Press, Beijing.

Yang H, Xu ZY, Lei MG, Li FE, et al. (2010). Association of 3 polymorphisms in porcine troponin I genes (TNNI1 and TNNI2) with meat quality traits. J. Appl. Genet. 51: 51-57.

Zuo B, Xiong YZ, Su YH, Deng CY, et al. (2004). Mapping of quantitative trait loci on porcine chromosome 7 using combined data analysis. Asian-Austr. J. Anim. Sci. 17: 1350-1354.

Zuo B, Yang H, Wang J, Lei MG, et al. (2007). Molecular characterization, sequence variation and association with fat deposition traits of ACOX1 gene in pigs. J. Anim. Feed Sci. 16: 433-444. 